\title{
Low-grade waste heat driven desalination technology
}

\author{
Alexander Christ ${ }^{1,2}$, Xiaolin Wang ${ }^{3}$, Klaus Regenauer-Lieb ${ }^{2}$, and Hui Tong Chua ${ }^{1,4, *}$ \\ 1 School of Mechanical and Chemical Engineering, The University of Western Australia, 35 Stirling Hwy, Crawley WA 6009, Australia \\ 2 School of Earth and Environment, The University of Western Australia, 35 Stirling Hwy, Crawley WA 6009, Australia \\ 3 School of Engineering, The University of Tasmania, Hobart TAS 7001, Australia \\ 4 School of Environmental Science and Engineering, Taiyuan University of Technology, Taiyuan, Shanxi Province, P.R. China
}

Received 27 August 2012 / Accepted 13 November 2012 / Published online 4 February 2014

\begin{abstract}
Low-grade heat driven multi-effect distillation (MED) desalination is a very promising environmentally friendly, low emission technology. Many countries, such as Australia, are water short and conventional desalination technology is energy intensive. If a primary fossil fuel source is used, then desalination will significantly contribute to carbon dioxide emission. Low-grade waste heat from process plants and power plants generate minimal additional carbon dioxide. This source of energy is typically abundant at a temperature around $65-90{ }^{\circ} \mathrm{C}$, which dovetails with MED technology. In this paper, we report on a new MED technology that couples perfectly with low grade waste heat to give at least a $25 \%$ freshwater yield improvement compared with conventional MED design. Typical applications and their expected improvement will also be reported.
\end{abstract}

Key words: Distillation, MED, Waste heat, Low-grade heat, Sensible heat, Evaporator.

\section{Nomenclature}

BPE Boiling point elevation, ${ }^{\circ} \mathrm{C}$

$c_{\mathrm{p}} \quad$ Specific heat capacity, $\mathrm{kJ} / \mathrm{kgK}$

$L \quad$ Latent heat, $\mathrm{kJ} / \mathrm{kg}$

$\dot{m} \quad$ Mass flow rate, $\mathrm{kg} / \mathrm{s}$

$\dot{Q} \quad$ Heat transfer rate, $\mathrm{kW}$

$T, \vartheta \quad$ Temperature, ${ }^{\circ} \mathrm{C}$

$S \quad$ Salinity, $\mathrm{kg} / \mathrm{kg}$

SDM Single effect distillation module

\section{Subscripts}

\section{evap Evaporator}

$i \quad$ Effect or condenser input

$f \quad$ Feed

$h \quad$ Heating medium

hyd Hydrostatic head

$s \quad$ Saturated

$o \quad$ Effect or condenser outlet

$v \quad$ Vapour

$n \quad$ Number of effect

1 Hotter side of heat exchanger

2 Colder side of heat exchanger

$z \quad$ Final condenser

\section{Introduction}

In many countries scarcity of freshwater is a serious issue. Globally this problem is getting worse as populations grow, lifestyles in developing countries improve, and climate change is leading to locally distorted weather patterns. According to the World Health Organization almost one fifth of the world's population is living in areas where water is physically scarce [1]. Even in areas with significant rainfall and freshwater resources, the water demand for households, agriculture and industry can be a serious issue, particularly in regions with a high population density.

Industrial desalination of seawater or process water is one of the solutions to assist in alleviating this problem. Technically there are several possible ways to turn seawater into freshwater. However, the major technologies currently applied on an industrial scale can be split in two categories [2, 3]: (1) thermal processes including multi-stage flash distillation (MSF), multieffect distillation (MED), thermal vapour compression (TVC) and mechanical vapour compression processes (MVC) and (2) membrane processes including reverse osmosis (RO).

In general, the overall energy consumption of all the desalination processes is very high, and consequently energetic optimization is one of the key challenges in the field of desalination. This can be addressed by two different approaches: (1) optimization of the desalination technology in order to minimize the overall consumption of energy and 


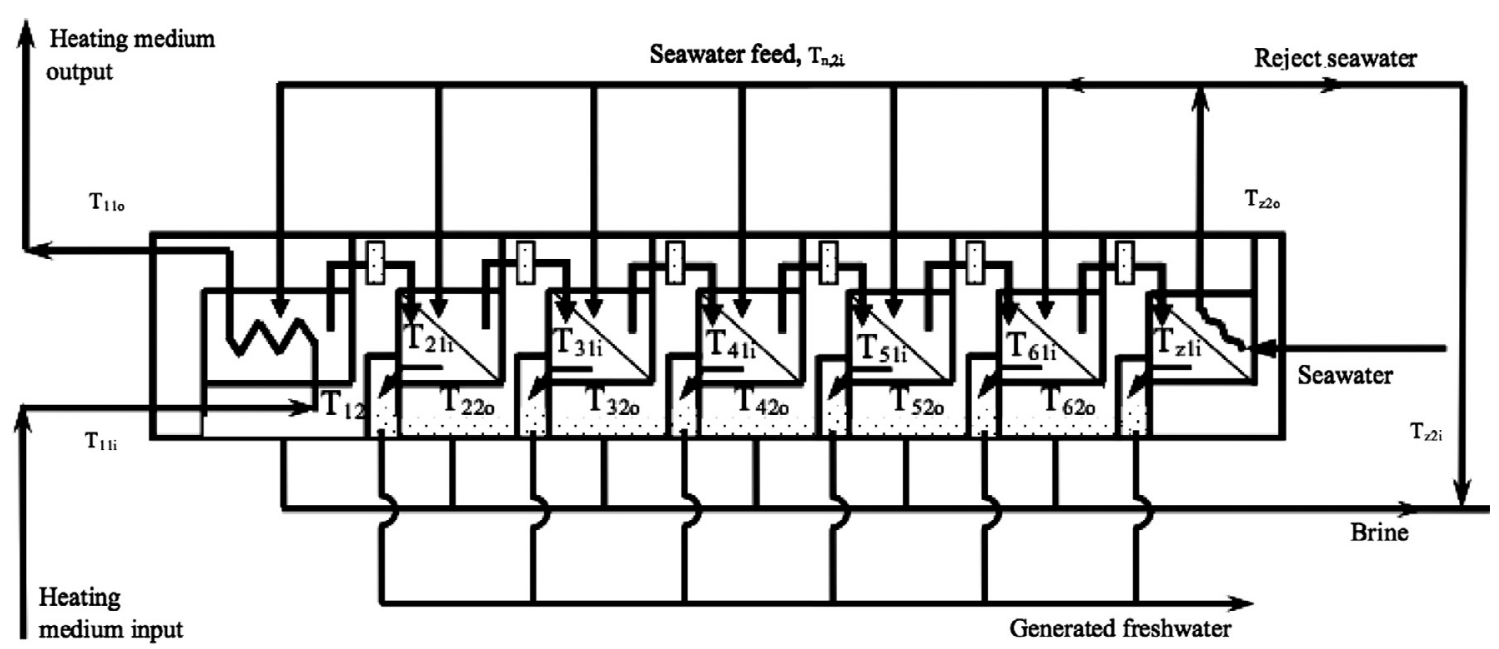

Figure 1. Schematic of a basic multi-effect-distillation system [4].

(2) utilization of energy sources with reduced cost and environmental impact, like renewable energies or waste heat. This paper presents the optimization of the MED technology combining both approaches for low-grade heat applications.

In general, thermal driven systems consume more energy than RO, but whereas the latter requires electrical power (which represents energy of a high quality), thermal systems can be operated with a broad range of heat sources. This makes them an optimal choice to utilise low-grade heat, including waste heat. However, current MED technologies for waste heat extraction are so far mainly focused on low pressure steam.

In contrast the novel MED design presented in this paper is particularly tailored for the use of sensible low-grade heat sources. Especially in the range of $65-100{ }^{\circ} \mathrm{C}$ a vast number of these sources are available, which cannot be optimally used by current technologies. Examples include cooling water from industrial processes, mining sites, power generation, and renewable energy sources like geothermal energy. In most of these cases the energy in this temperature range is currently unused, rejected to the environment, even causing additional expenses for cooling facilities. The proposed novel MED design allows to harvest this promising sources in an economic and environment friendly way.

This is achieved by a simulation based, comprehensive optimization approach considering the individual nature of the lowgrade heat source; for example geothermal heat.

\section{Working principle of a MED plant}

A basic MED plant (Figure 1) comprises a set of evaporators, called "effects", and a final condenser. The motive steam or hot water enters the heat exchanger of the first effect as the heating medium. Feedwater is applied on the opposite side of the heat exchanger. As the energy is transferred from the hotter heating medium to the colder feed, the feedwater warms up to its boiling temperature and a portion of it evaporates subsequently. The generated vapour passes through demisters and is condensed in the second effect, where the released latent heat of the condensation is utilized to evaporate more feedwater.
This process is repeated in several effects. To maintain the heat flow, each subsequent effect operates at diminishing temperature and pressure.

To condense the vapour generated in the last effect a final condenser cooled by seawater is used. By using parts of the heated up cooling water for the feed stream, a portion of the heat is recovered. The remaining portion of cooling water is rejected back to the sea together with the produced brine. The generated condensate in each effect consists of boiler grade freshwater and can be used for the desired purpose.

Based on the basic MED, several design variations are possible: for example, thermal vapour compression to increase efficiency, treatment of contaminated water rather than saline water and optimization to minimize waste water flow.

\section{Numerical analysis of the MED system}

The main focus of this article is to show the new design and its improvement over the conventional systems rather than the simulation model itself. Therefore only the basics of the model are presented below. The model is developed based on the steady state mass and energy balances coupled with the heat transfer equations for each individual effect together with the ratio between the mass of feedwater to that of produced freshwater.

When only sensible heat is used as the heating medium, the energy balance for the first effect (evaporator) can be expressed as:

$$
\begin{aligned}
\dot{Q}_{\text {evap }, i} & =\dot{m}_{h, i} c_{p, h}\left(T_{11 i}-T_{11 o}\right) \\
& =\dot{m}_{1, f} c_{p, f}\left(T_{12 o}-T_{12 i}\right)+\dot{m}_{1 v} L_{12},
\end{aligned}
$$

$c_{p, h}$ and $c_{p, f}$ are the constant pressure specific heat capacities of the heating medium and the feed, respectively; $L_{12}$ is the latent heat of evaporation, which depends on the boiling temperature and can be calculated by the vapour saturation temperature [5]:

$$
L=2499.5698-2.204864 \vartheta_{v s}-1.596 \times 10^{-3} \vartheta_{v s}{ }^{2},
$$


where $\vartheta_{v s}$ the vapour saturation temperature in ${ }^{\circ} \mathrm{C}$. The vapour saturation temperature is a function of the pressure in the evaporator vapour space. It is less than the boiling temperature $T_{12}$ by the boiling point elevation:

$$
T_{12}=T_{v s}+\mathrm{BPE}+\Delta T_{\mathrm{hyd}}
$$

$\Delta T_{\text {hyd }}$ is the boiling point rise causes by the hydrostatic head in the effect. This term is negligible in horizontal falling film evaporators. The boiling point elevation BPE reflects the increase in the boiling temperature due to the salts dissolved in the water. It can be calculated based on the following empirical relation $[6,7]$ :

$$
\begin{gathered}
\mathrm{BPE}=A S^{2}+B S, \\
A=-4.584 \times 10^{-4} \vartheta^{2}+2.823 \times 10^{-1} \vartheta+17.95, \\
B=1.536 \times 10^{-4} \vartheta^{2}+5.267 \times 10^{-2} \vartheta+6.56,
\end{gathered}
$$

where $S$ is the salinity in $\mathrm{kg} / \mathrm{kg}$ and $\vartheta$ the boiling temperature in ${ }^{\circ} \mathrm{C}$. This formula is valid for $0 \leq S \leq 0.12 \mathrm{~kg} / \mathrm{kg}$ and $0 \leq \vartheta \leq 200^{\circ} \mathrm{C}$.

Since the vapour generated in the first effect acts during condensation as heat source for the subsequent effect, the energy balance for the $n$th effect can be expressed as:

$$
\dot{Q}_{n, i}=\dot{m}_{n-1, v} L_{n, 1}=\dot{m}_{n, f} c_{p, f}\left(T_{n, 1 o}-T_{n, 1 i}\right)+\dot{m}_{n v} L_{n 2} .
$$

It must be mentioned that in real processes additional effects lead to a further reduced vapour temperature besides the boiling point elevation. These include non-equilibrium effects, temperature losses, as well as pressure related drops caused by the demister and friction losses. However, these are typically very small and their effect on the vapour temperature drop can be neglected for a simple model. Consequently the input temperature of the vapour can be assumed as the boiling temperature from the vapour outlet of the upstream effect.

By far the largest amount of the initial input energy is carried over in the form of latent heat from effect to effect. Only a small amount of sensible heat is carried out with the brine and freshwater streams leaving each effect. Therefore the effects do downgrade the energy, but consume only a small portion. Consequently the production in each subsequent effect is only slightly reduced. On the other hand, this requires a rejection of the major part of energy in the final condenser by cooling with seawater. The energy balance for the final condenser can be calculated:

$$
\dot{Q}_{z=n, i}=\dot{m}_{n-1, v} L_{z, 1}=\dot{m}_{z, 2 i} c_{p f}\left(T_{z, 2 o}-T_{z, 2 i}\right),
$$

whereas $\dot{m}_{z, 2 i}$ is the cooling water stream and $T_{z, 2 i}$ the cooling water input temperature, which is in typical applications the sea water temperature. Since a portion of the cooling water output stream is used as feed, the cooling water output temperature $T_{z, 2 o}$ determines the feed water temperatures for each effect $\left(T_{n, 2 i}\right)$. In an idealized system, $T_{n, 2 \mathrm{i}}=T_{z, 2 o}$ can be assumed.

Typically, the major optimization aim is to maximize freshwater production from a given initial heat input. This can be expressed as:

$$
\sum_{n=1}^{Z-1} \dot{m}_{n, v}=\max !
$$

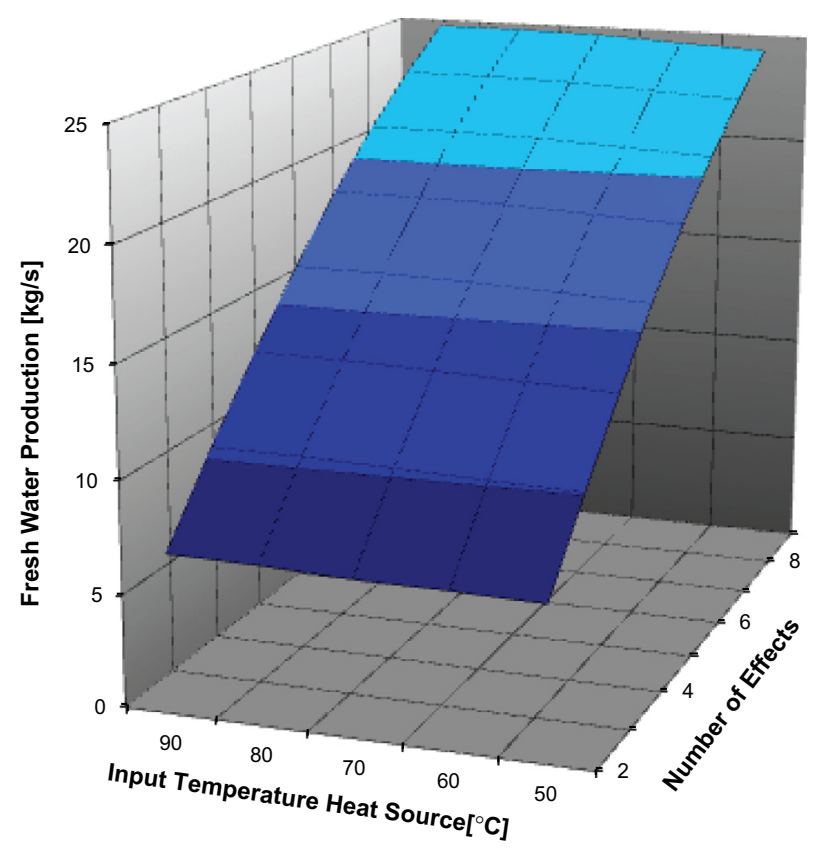

Figure 2. Dependency of the freshwater production on the heat source input temperature and the number of effects, shown for an example standard MED.

Boundary conditions are formed by the laws of thermodynamics, with additional technical, economic, and operational constraints. These include for example minimal temperature differences in the heat exchanger and environmental compliance.

The model is calibrated against the plant performance data of a commercially available Alfa Laval single-effect distillation unit [8]. Also a modified simulation version incorporating the new design presented below has been developed.

\section{Conventional MED systems}

In most of the current applications, the fuel costs to operate the system are proportional to the energy input, $\dot{Q}_{\text {evap }, i}$. These cost can be of a direct or indirect nature, for example costs for the fuel to heat the system directly or production losses of electrical energy from power plants due to the extraction of low pressure steam from the Rankine cycle. Because of the high energy demand, the associated fuel costs are major drivers for the costs of desalination, in particular in the background of increasing energy costs. Consequently, the major optimization aim is traditionally minimal energy consumption per unit freshwater.

Figures 2 and 3 display the results of the simulation software based on an example standard MED, in this case driven by a liquid heat source. For comparison reasons the heat input is kept constant. The significant impact of the number of effects on the freshwater generation is shown in Figure 2 and consequently the impact on the specific energy consumption is shown in Figure 3. This is due to the fact that most of the energy is carried over via the latent heat of the vapour, whereas only a small amount is actually rejected from the process by the brine 


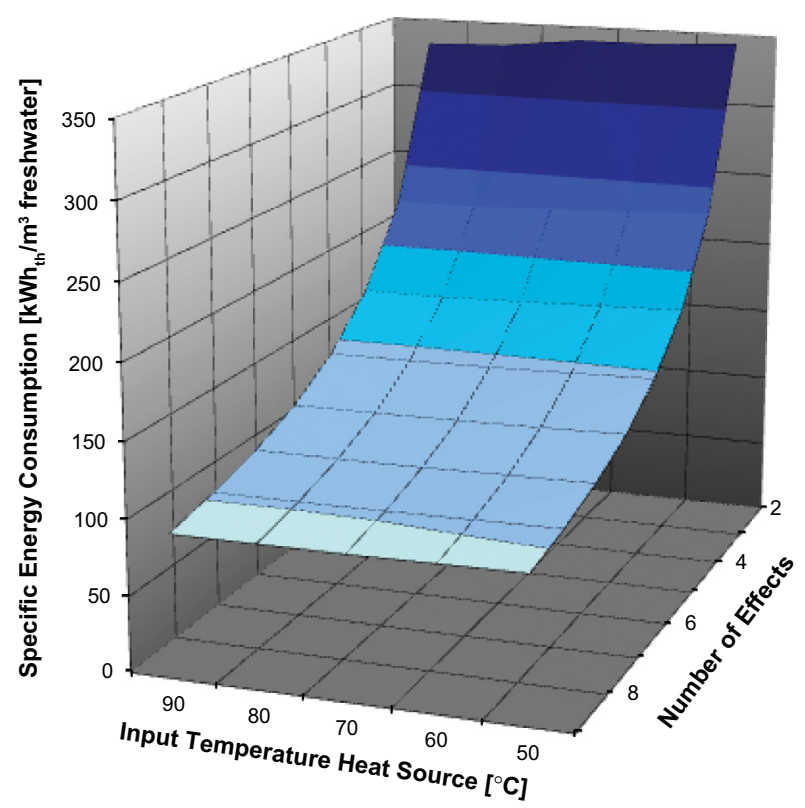

Figure 3. Dependency of the specific energy consumption per $\mathrm{m}^{3}$ freshwater on the heat source input temperature and the number of effects, shown for an example standard MED.

and freshwater stream. As it can also be shown, the heat source temperature itself has only minor influence on the production of at a given number of effects, provided the heat input is constant.

Nonetheless the temperature profile of the heat source is a significant parameter of the system design since it determines the top end of the available temperature interval and is therefore of material importance for the maximal possible number of placed effects. The lower end of the temperature interval is defined by the cooling water temperature, usually the seawater temperature. The interval can be split by the number of effects, whereas the minimal temperature difference over each effect is primarily limited by economic considerations to $1.5-3{ }^{\circ} \mathrm{C}$ $[9,10]$.

Most current MEDs are steam driven, where the isothermal nature of the condensation allows the brine temperature in the hottest effect, the so-called "top brine temperature", to approach the heat source temperature. A different case applies if only sensible heat is used and the heating medium undergoes a temperature change: restricted by the second law of thermodynamic, the top brine temperature is depending on this temperature gradient (pinch-point-analysis). As a result, any increase of the applied temperature difference of the heating medium is reducing the available temperature range to place effects, and therefore the efficiency of the system.

In this context it must be mentioned that in MED systems without additional pretreatment like nanofiltration (NF) the top brine temperature is limited by scaling issues to $65-70{ }^{\circ} \mathrm{C}$ [11].

\section{Novel MED design}

For true waste heat sources and most renewable energies the direct relationship between the energy input $\dot{Q}_{\text {evap }, i}$ and fuel costs does not apply. This includes all situations where the energy costs are not a function of the actual amount of energy used. A typical example is geothermal energy: costs come from the well construction, the energy demand of the production pumps, etc. to make the hot groundwater available at the surface, but these costs are totally independent from the energy that is finally extracted from that water.

The same applies for several waste heat applications: the energy content of hot water from 65 to $100{ }^{\circ} \mathrm{C}$ is too low for most thermodynamic processes. It has therefore to be rejected unused to the environment, causing additional expenses for cooling facilities. In consequence, this low-grade heat can frequently be available at neutral cost.

As a result, the efficiency does not solely depend on the minimal energy requirement per unit freshwater anymore, rather the capability to extract as much energy as possible. Hence a paradigm shift is required. Instead of an optimization of the desalination unit alone, the entire low grade heat system with its individual characteristics must be considered. Instead of the minimal energy consumption of the desalination unit, the key objective is to maximize freshwater yield from the available low grade heat source.

Figure 4 shows the novel MED design incorporating this optimization approach. The hot water is first introduced into the first effect heat exchanger as the driving source for the primary MED plant. The hot water leaving the heat exchanger is still very hot. Therefore this water is pumped into a single effect distillation module (SDM) where a portion of vapour is generated. This generated vapour is judiciously fed into an appropriate effect within the primary MED plant. Obviously, the size and seawater feed rate of the following effects have to be increased accordingly to cater for the boost in capacity. It is obvious that this process substantially boosts the freshwater yield and results in significantly increased exploitation of the low grade heat source. Alternative options consisting of two independent conventional MED in series could reach to a similar result but would be far more expensive.

\section{Expected improvements}

To determine the expected improvements for particular process conditions, the simulation model is used.

Figures 5 and 6 depict the improvement of the proposed design applied respectively to a four- and a six-effect distillation plant at different heat source and cooling water temperatures. This reflects typical operating ranges for commercially available multi-effect distillation plants at a heat source temperature between 75 and $90{ }^{\circ} \mathrm{C}$.

The corresponding operating conditions are listed in Table 1. As it can be shown, the proposed design improves the freshwater yield by $25-60 \%$ depending on the heat source and cooling water temperatures. It is observed from the figures that the improvement using the SDM design decreases with the heat source temperature. With higher heat source temperature, a higher temperature drop in the first effect of the primary plant can be realised. Related to the total energy input, the proportion of recovered energy is therefore lower than that for a lower temperature heat source and hence the improvement to freshwater yield diminishes. 


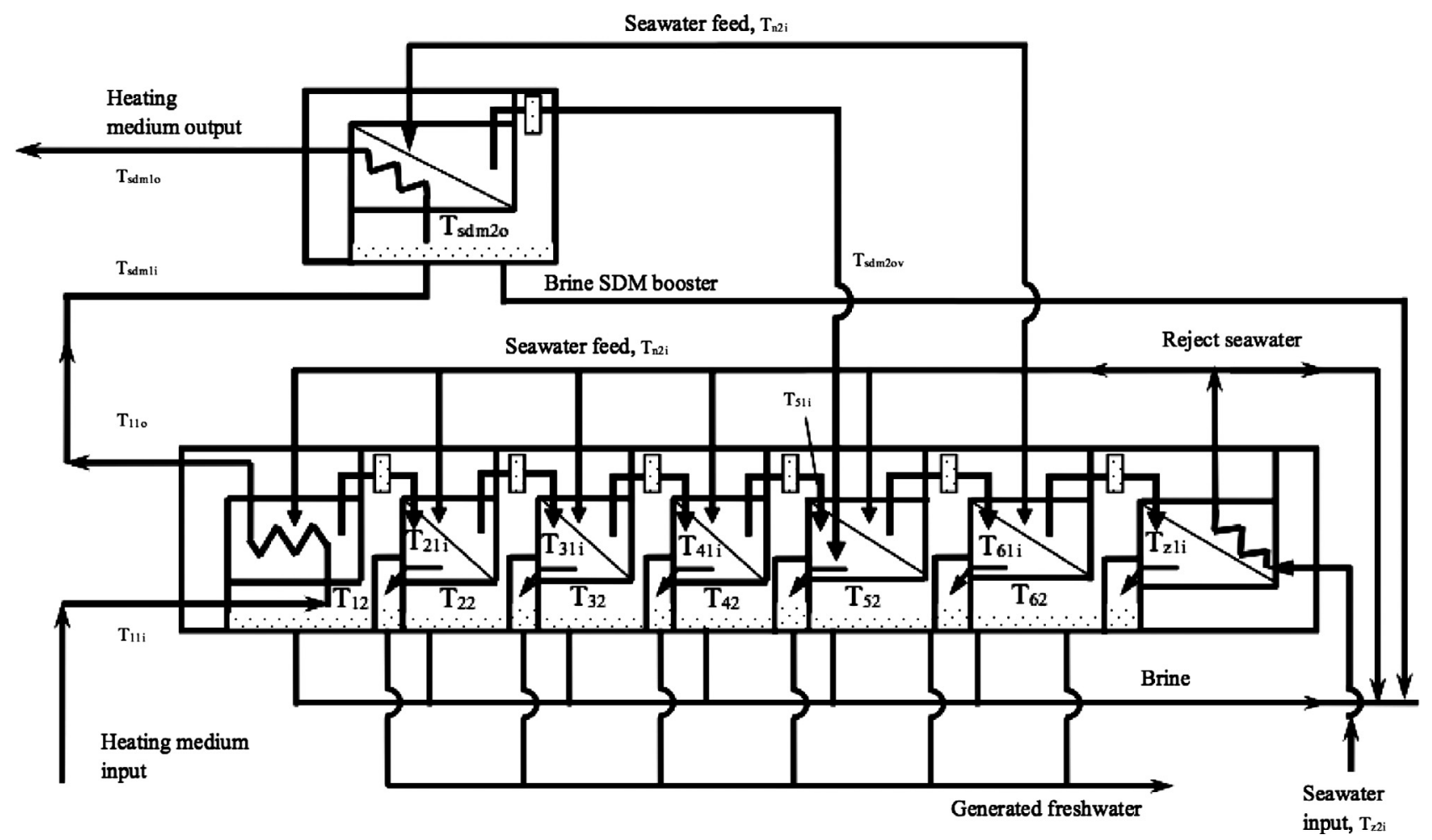

Figure 4. Schematic of the advanced multi-effect-distillation with single-effect-module booster [4].

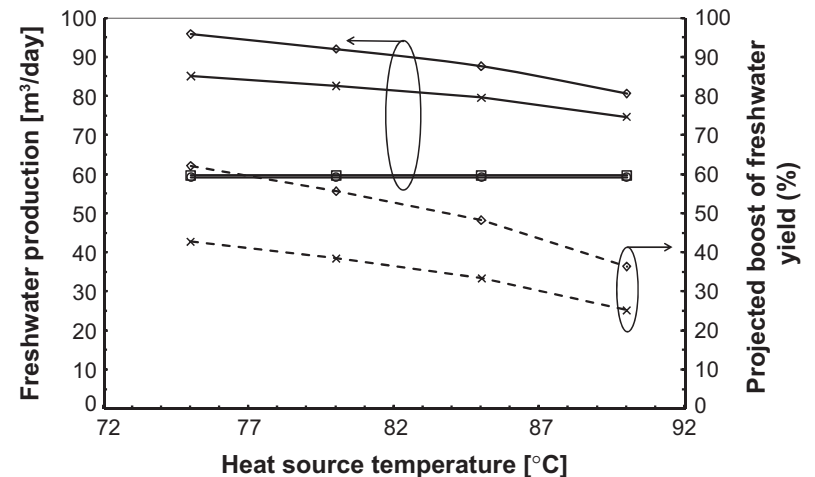

Figure 5. The efficacy of the novel MED design applied to a foureffect distillation plant. $\bigcirc$, freshwater yield in a standard four-effect distillation plant at a cooling water temperature of $25^{\circ} \mathrm{C}$; $\square$, freshwater yield of a standard four-effect distillation plant at a cooling water temperature of $32{ }^{\circ} \mathrm{C}$. $\diamond$, freshwater yield and improvement by using our proposed design at $25{ }^{\circ} \mathrm{C} ; \times$, freshwater yield and improvement by using the proposed design at $32{ }^{\circ} \mathrm{C}$ [4].

For comparison reasons, the influence of the cooling water temperature is also shown. It is notable that for a fixed number of effects the boost decreases with increasing cooling water temperature. At a lower cooling water temperature, the whole MED plant can operate at a relatively lower temperature in each of the effects and hence the vapour generated in the single seawater distillation module can be fed into a higher effect, thereby generating more freshwater through the various effects

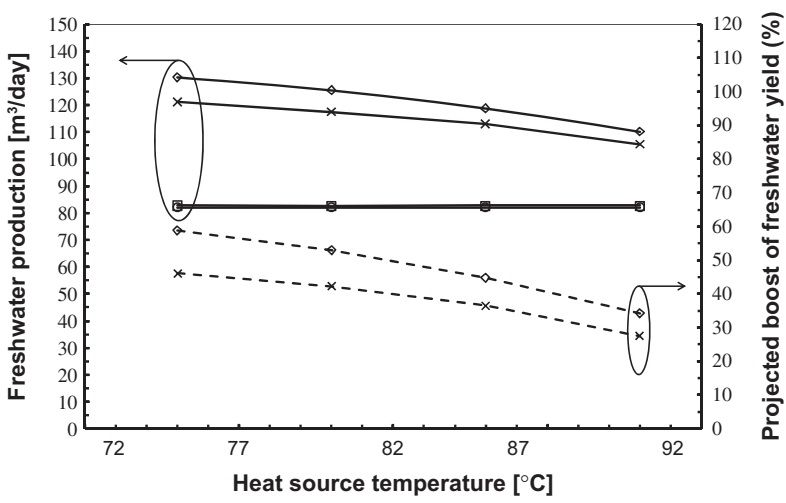

Figure 6. The efficacy of the proposed scheme as applied to a sixeffect distillation plant. $\bigcirc$, freshwater yield in a standard four-effect distillation plant at a cooling water temperature of $25^{\circ} \mathrm{C} ; \square$, freshwater yield of a standard four-effect distillation plant at a cooling water temperature of $32{ }^{\circ} \mathrm{C}$. $\diamond$, freshwater yield and improvement by using our proposed design at $25{ }^{\circ} \mathrm{C} ; \times$, freshwater yield and improvement by using our proposed design at $32{ }^{\circ} \mathrm{C}$ [4].

downstream. For example when the cooling water temperature is $32{ }^{\circ} \mathrm{C}$, the vapour generated in the SDM can only be fed into the last effect of a four effect distillation plant and the fourth effect of a six effect distillation plant. However when the cooling water temperature is $25^{\circ} \mathrm{C}$, the whole MED plant operates at a relatively lower temperature in each effect. The vapour generated in the SDM can now be fed into the third effect of the four effect distillation plant and the third effect of the six effect 
Table 1. The operating conditions for the four and six effect distillation plants.

\begin{tabular}{|c|c|c|c|c|c|c|}
\hline$T_{11 i}$ & $T_{11 \mathrm{o}}$ & $\dot{Q}_{\text {evap }, i}$ & $T_{\mathrm{sdm}, i}$ & $T_{\mathrm{sdm}, o}$ & $\dot{Q}_{\mathrm{sdm}, i}$ & $\dot{m_{h}}$ \\
\hline${ }^{\circ} \mathrm{C}$ & ${ }^{\circ} \mathrm{C}$ & $\mathrm{kW}$ & ${ }^{\circ} \mathrm{C}$ & ${ }^{\circ} \mathrm{C}$ & $\mathrm{kW}$ & $\mathrm{kg} / \mathrm{s}$ \\
\hline \multicolumn{7}{|c|}{ MED without SDM } \\
\hline 80 & 68 & 450.0 & - & - & - & 9.0 \\
\hline 85 & 70 & 450.0 & - & - & - & 7.2 \\
\hline 90 & 70 & 450.0 & - & - & - & 5.4 \\
\hline 80 & 68 & 450.0 & 68 & 59.5 & 318.8 & 9.0 \\
\hline 85 & 70 & 450.0 & 70 & 60.8 & 276.0 & 7.2 \\
\hline 90 & 70 & 450.0 & 70 & 60.8 & 207.0 & 5.4 \\
\hline
\end{tabular}

Cooling temperature, $T_{z, i} 25^{\circ} \mathrm{C} / 32{ }^{\circ} \mathrm{C}$

Salt concentration $\quad 35,000 \mathrm{ppm}$

distillation plant. The recovered energy is then used to generate more freshwater. Therefore a greater improvement exists at lower cooling temperatures.

\section{Possible applications}

Possible applications of the novel design include all cases where sensible (waste) heat is available. Economical operation can typically be achieved from a heat source input temperatures of $\sim 65{ }^{\circ} \mathrm{C}$ or greater.

This includes, but is not limited to:

- Geothermal systems;

- Waste heat from power generation;

- Waste heat from industrial processes;

- Waste heat from oil and mining sites;

- Industrial laundries.

It is anticipated that some existing MED can be retrofitted with the new system. This applies to small and midsize units in particular with plate type heat exchangers, where the shell design allows an increase in heat exchanger surface for the effects after the booster injection.

\section{Conclusion}

An advanced design paradigm for geothermal and waste heat driven MED plants is proposed. Combined with true waste heat sources, an improvement of the freshwater yield by $25 \%$ to $60 \%$ over standard MED can be expected. This is achieved by a cross disciplinary approach also considering the individual nature of the heat source. The improved process allows a cost effective, environmental friendly desalination with a minimal carbon footprint by the use of broadly available sensible waste heat in the range of $\sim 65-100{ }^{\circ} \mathrm{C}$.

In general, the actual expected improvement over a standard MED is very case dependent. For an optimal result, the specific nature of the present heat source must be taken into account.
However, the final improvement is in particular a function of the proportion of the energy input in the basic MED versus the input in the SDM part. Typical applications commonly allow a minimal improvement of at least $25 \%$, with values ranging up to $65 \%$ or more. Further simulation results are published in [11].

Acknowledgements. We gratefully acknowledge the financial support from the Western Australian Geothermal Centre of Excellence and the National Centre of Excellence in Desalination Australia, which is funded by the Australian Government through the National Urban Water and Desalination Plan.

\section{References}

1. World Health Organization. 2009. 10 facts about water scarcity. http://www.who.int/features/factfiles/water/en/index

2. El Saliby I. 2009. Desalination plants in Australia, review and facts. Desalination, 247(1-3), 1-14.

3. Miller JE. 2003. Review of water resources and desalination technologies. Sandia National Laboratories: Albuquerque.

4. Wang X. 2011. Low grade heat driven multi-effect distillation technology. International Journal of Heat and Mass Transfer, 54(25-26), 5497-5503.

5. El-Dessouky H. 1998. Steady-state analysis of the multiple effect evaporation desalination process. Chemical Engineering \& Technology, 21(5), 437-451.

6. Bromley LA. 1974. Thermodynamic properties of sea salt solutions. AIChE Journal, 20(2), 326-335.

7. Sharqawy MH, John VJH, Zubair SM. 2012. Thermophysical properties of seawater: a review of existing correlations and data. Desalination and Water Treatment, 44(1-3), 361-361.

8. Alfa Laval Marine \& Diesel Product Catalogue. 2003. Desalt JWP-16-C Series. Lund, Sweden: Alfa Laval.

9. Ophir A, Lokiec F. 2005. Advanced MED process for most economical sea water desalination. Desalination, 182(1-3), 187-198.

10. Awerbuch L. 2012. Understanding of thermal distillation processes. IDA Academy: Singapore.

11. Awerbuch L. 2012. Understanding of hybrid systems and dual purpose power desalination. IDA Academy: Singapore.

Cite this article as: Christ A, Wang X, Regenauer-Lieb K \& Chua H: Low-grade waste heat driven desalination technology. Int. J. Simul. Multisci. Des. Optim., 2014, 5, A02. 